\title{
RESEARCH
}

\section{miR17-92 cluster drives white adipose tissue browning}

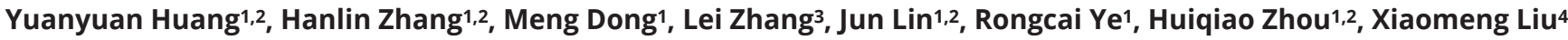 \\ and Wanzhu Jin ${ }^{1}$ \\ ${ }^{1}$ Key Laboratory of Animal Ecology and Conservation Biology, Institute of Zoology, Chinese Academy of Sciences, Beijing, China \\ 2The University of the Chinese Academy of Sciences, Beijing, China \\ ${ }^{3}$ Center for Advanced Biomedical Imaging and Photonics, Beth Israel Deaconess Medical Center, Harvard University, Boston, Massachusetts, USA \\ 4Institute of Neuroscience and Translational Medicine, College of Life Science and Agronomy, Zhoukou Normal University, Zhoukou, China
}

Correspondence should be addressed to X Liu or W Jin: Ixmxm_99@126.com or jinw@ioz.ac.cn

\begin{abstract}
White adipose tissue (WAT) browning may have beneficial effects for treating metabolic syndrome. miRNA are important regulators of the differentiation, development, and function of brown and beige adipocytes. Here, we found that the cold-inducible miRNA17-92 cluster is enriched in brown adipose tissue (BAT) compared with WAT. Overexpression of the miR17-92 cluster in C3H10T1/2 cells, a mouse mesenchymal stem cell line, enhanced the thermogenic capacity of adipocytes. Furthermore, we observed a significant reduction in adiposity in adipose tissue-specific miR17-92 cluster transgenic (TG) mice. This finding is partly explained by dramatic increases in white fat browning and energy expenditure. Interestingly, the miR17-92 cluster stimulated WAT browning without altering BAT activity in mice. In addition, when we removed the intrascapular BAT (iBAT), the TG mice could maintain their body temperature well under cold exposure. At the molecular level, we found that the miR17-92 cluster targets Rb1, a beige cell repressor in WAT. The present study reveals a critical role for the miR17-92 cluster in regulating WAT browning. These results may be helpful for better understanding the function of beige fat, which could compensate for the lack of BAT in humans, and may open new avenues for combatting metabolic syndrome.
\end{abstract}
Key Words
- brown adipose tissue
- miR17-92 cluster
- beige adipocyte
- $\mathrm{Rb} 1$

\section{Introduction}

Unlike the function of WAT, the mitochondria in BAT consume large amounts of fuel directly from the lipid pool for heat generation (Lowell \& Spiegelman 2000, Rosen \& Spiegelman 2014). In addition, accumulating evidences have also demonstrated a new subset of cells in WAT, called beige or brite cells, which are similar to classic BAT in morphology and function; these cells have been suggested to have strong antiobesity and antidiabetic benefits (Cypess et al. 2009, Barbatelli et al. 2010, Petrovic et al. 2010, Wu et al. 2012). Cold temperatures and drugs stimulate the appearance/recruitment of beige cells to white adipose depots (Kalinovich et al. 2017, Yuan et al. 2017). Past studies have shown that infrequent functional brown adipocytes also exist in adult humans. Importantly, a negative correlation was observed between the recruitment of beige cells and BMI. This finding suggests that beige cells may critically inhibit obesity and obesity-related metabolic diseases in humans (Cypess et al. 2009, Saito et al. 2009).

miRNAs are one of many factors identified to be effective in activating beige fat (Trajkovski et al. 2012, 
Chen et al. 2013, Sun \& Trajkovski 2014). miRNAs are small (21-23 nucleotides), noncoding, highly conserved endogenous RNAs that regulate gene expression (Tanzer $\&$ Stadler 2004). Many miRNAs, such as the BAT-enriched miR193b-365 (Mori et al. 2014), miR155 (Chen et al. 2013), and miR133, which targets Prdm16 (Yin et al. 2013), and the WAT-enriched miR143 (Jordan et al. 2011), have been reported to directly or indirectly modify browning or differentiation. These miRNAs have been characterized as potential targets for the treatment of diabetes. The miR17-92 cluster consists of six miRNAs: miR17-5p, miR18a-5p, miR19a-3p, miR20a-5p, miR19b-3p, and miR92a-3p (Mendell 2008). A previous study showed that stable transfection of 3T3-L1 cells with the miR17-92 cluster resulted in lipid depot accumulation through targeting of $\mathrm{Rb} 2$ (Wang et al. 2008). The retinoblastoma susceptibility protein $(\mathrm{Rb})$ family includes Rb1, P107 and P130 (Classon \& Dyson 2001), which play important roles during fat development and adipogenesis (Scime et al. 2005), and mice with $\mathrm{Rb} 1$ haploinsufficiency showed reduced dietinduced obesity, insulin resistance, and hepatic steatosis (Mercader et al. 2009). Mice with repressed Rb1 expression also showed browning in WAT (Wang et al. 2013).

Thus, in the present study, we identified key regulators and showed that the miR17-92 cluster can increase energy expenditure, maintain body temperature well under cold exposure and decrease adiposity via the activation of mitochondrial activity by targeting Rb1. This cluster also acts as an important regulator of browning and thermogenic function independent of BAT activity.

\section{Materials and methods}

\section{Animals}

Protocols of animal studies were approved by the Institutional Animal Care and Use Committee (Institute of Zoology, Chinese Academy of Sciences). All studies used male C57BL/6 J mice that were purchased from Vital River Laboratory Animal Technology (Beijing, China).

The miR17-92 cluster DNA sequence was subcloned into a pBluescript vector containing a $5.4 \mathrm{~kb}$ adiponectin promoter (Wang et al. 2010), which was used to inject into fertilized embryos harvested from C57BL/6 J mice to get transgenic miR17-92 cluster TG mice. DNA samples from tail clips of subsequent litters were checked by PCR with primers in Table 1 that amplified an 300-bp fragment in positive samples (data not shown).

\section{Antibodies}

The following antibodies were used in this study: antiUCP1 (Abcam, ab10983; 1:1000), anti-OXPHOS (Abcam, ab110413; 1:500), anti-PPAR 2 (CST, 2443; 1:1000), anti-Rb1 (Abcam, ab24; 1:500), anti-GAPDH (CST, 2118S; $1: 2000)$ and anti- $\beta$-actin (Sigma, A2228; 1:5000).

\section{RNA sequencing of plasma}

Eight-week-old C57BL/6J mice were randomly divided into two groups ( $n=12$ in each group). One group was exposure at $4^{\circ} \mathrm{C}$ for 2 weeks, and the control group was kept in the room temperature $\left(25^{\circ} \mathrm{C}\right)$. Plasma of both groups was

Table 1 Primer sequences.

\begin{tabular}{l} 
Primer \\
\hline AP2 \\
CEBP $\alpha$ \\
CEBP $\beta$ \\
Cidea \\
Cyclophilin A \\
PGC1 $\alpha$ \\
PPAR 2 \\
PRDM16 \\
Tfam \\
UCP1 \\
Rb1 \\
Pri-miR17 \\
Pri-miR18a \\
Pri-miR19a \\
Pri-miR19b \\
Pri-miR20a \\
Pri-miR92a \\
COX II \\
$\beta$-Globin
\end{tabular}
Forward
GAAGACTGCGAGGACCTC
GCGGGAACGCAACAACATC TGACGCAACACACGTGTAACTG TGCTCTTCTGTATCGCCCAGT CAAATGCTGGACCAAACACA ACAGCTTTCTGGGTGGATT TCGCTGATGCACTGCCTATG GAAGTCACAGGAGGACACGG GTCCATAGGCACCGTATTGC GGCAAAAACAGAAGGATTGC ACCTGATAACCTTGAACCTGC GAAGCTGTGACCAGTCAGAATAA GCACTGGCTGCGTCCAGTCG AGTTATGTCCTCATCCAATC GTCCTGTTATTGAGCACTGG GTGGATGTAGAGCCTGCGTG CTACACAGGTTGGGATTTG GCCGACTAAATCAAGCAACA GAAGCGATTCTAGGGAGCAG

\begin{tabular}{l}
\hline Reverse \\
\hline GAAGTGCCGTAATCCCCACAC \\
GTCACTGGTCAACTCCAGCAC \\
AACAACCCCGCAGGAACAT \\
GCCGTGTTAAGGAATCTGCTG \\
GCCATCCAGCCATTCAGTCT \\
TGAGGACCGCTAGCAAGTTT \\
GAGAGGTCCACAGAGCTGATT \\
CTCGCTCCTCAACACACCTC \\
CCCATGCTGGAAAAACACTT \\
TAAGCCGGCTGAGATCTTGT \\
GCTTGTGTCTCTGTATTTGCAG \\
GAGGCAGCTGTCAGCATAAT \\
CAGAGGGCTGCAAACACAAC \\
CGCAGGCTCTACATCGACAC \\
CCATACAGAGAAACACAGCA \\
AGTTGGCTGGGCGATGGCCA \\
TCACAGTTTACAGAATGTGT \\
CAATGGGCATAAAGCTATGG \\
GGAGCAGCGATTCTGAGTAG
\end{tabular}


collected at the same time and miRNA in plasma extracted by the Tiangen miRcute miRNA extraction and separation kit. To improve the accuracy of sequencing, A total of $200 \mu \mathrm{L}$ plasma was extracted from each sample. The obtained samples were entrusted to Ribobio Co. Ltd (Ribobio, China) for detection. Due to the very low concentration of miRNA, the samples from the same groups were remixed for a single sample to for secondgeneration sequencing analysis.

To make a more intuitive comparison between the room temperature and cold exposure, the raw data reads were uploaded and calculated into Gene Cluster 3.0 with similarity matrix of Speakman Rank Correlation after log $(\mathrm{X} / 100)$ fetch, and the final graph was sharpened by Java Tree view.

\section{Real-time PCR}

Cells and tissues were extracted with TRIzol (Invitrogen), and cDNA was then reverse transcribed using M-MLV (Promega). Real-time PCR was performed using appropriate primer pairs to determine the relative gene expressions. The sequences of primers for the Real-Time PCR System are provided in Table 1 .

\section{Luciferase reporter assays}

The WT and mutated Rb1 3'-UTRs were cloned and inserted into the SacI and HindIII sites of the pMIRReport vector (Ambion). The mutated sites in the $\mathrm{Rb} 1$ 3'-UTR were designed as follows: WT 5'-GCACTTT-3' to mut1 5'-ATGTCCC-3' and WT 5'-CACTTT-3' to mut2 5'-TGTCCC-3'. HEK293T cells were co-transfected with pMIR-Report or WT or mutant pMIR-Report-Rb13'-UTR along with the PCDH-miR17-92 cluster using Lipofectamine 2000 (Invitrogen). Luciferase activity was measured using a luciferase activity assay kit (Promega, E1910). Renilla luciferase activity was used for normalization to determine the transfection efficiency.

\section{Cell transfection and differentiation}

C3H10T1/2 cells and 3T3-L1 cells were purchased from the National Platform of Experimental Cell Resources (Sci-Tech, Shanghai, China). C3H10T1/2 cells were transfected with PCDH or miR17-92 cluster and then differentiated according to the brown fat differentiation method (Yuan et al. 2017). Fully differentiated C3H10T1/2 cells were used in all experiments on day 6, and 3T3-L1 cells were transfected with PCDH or the miR17-92 cluster when cells were confluent and then treated with brown adipogenic induction cocktails (DMEM containing 10\% FBS, $1 \mu \mathrm{g} / \mathrm{mL}$ insulin, $1 \mu \mathrm{M}$ dexamethasone, $0.5 \mathrm{mM}$ isobutylmethylxanthine, $0.12 \mathrm{mM}$ indomethacin, and $1 \mathrm{nM} \mathrm{T3)}$ for the first 2 days. The medium was then replaced by medium supplemented with only insulin and T3, which was changed every other day. The cells were used in all experiments on day 10.

\section{Oxygen consumption assays}

C3H10T1/2 cells were overexpressed with PCDH or the miR17-92 cluster plasmids and then seeded onto 96-well XF96 cell culture microplates. After further differentiated for 6 days according to the brown fat differentiation method, XF24-3 extracellular flux analyzer (Agilent Technologies) was used to measure $\mathrm{O}_{2}$ consumption of differentiated adipocytes on day 6 , the cells were then treated with $10 \mu \mathrm{M}$ oligomycin (Sigma) to measure the ATP turnover. The maximum respiratory capacity was assessed after $1 \mu \mathrm{M}$ FCCP (Sigma) stimulation. Finally, mitochondrial respiration was blocked with $1 \mu \mathrm{M}$ rotenone (Sigma) and the residual oxygen consumption rate (OCR) was considered as non-mitochondrial respiration.

At 20 weeks, the WT and TG mice were assessed for oxygen consumption with a TSE Lab Master system, as previously described (Yuan et al. 2017, Zhao et al. 2020).

\section{Measurement of the mitochondrial DNA copy number}

Total DNA was isolated from BAT and SUB adipose tissue from the WT and TG mice at 21 weeks of age according to the manufacturer's instructions (Tiangen). The COX II gene was used to assess mitochondrial DNA copy number, and the $\beta$-globin gene was used for genomic reference. The primers used in the Real-Time PCR System are shown in Table 1.

\section{Oil red $O$ staining}

Fully differentiated cells were fixed with $4 \%(\mathrm{w} / \mathrm{v})$ formaldehyde for $30 \mathrm{~min}$ at room temperature and then stained with a $0.3 \%(\mathrm{w} / \mathrm{v})$ Oil Red O solution as described in our recent paper (Yuan et al. 2017). 


\section{Metabolic rate and physical activity}

Oxygen consumption and physical activity were determined with a TSE lab master. Mice were acclimated to the system for 20-24 h, and then $\mathrm{Vo}_{2}$ and $\mathrm{VcO}_{2}$ were measured during the next $24 \mathrm{~h}$. The animals were maintained at $24^{\circ} \mathrm{C}$ in a $12 \mathrm{~h}$ light: $12 \mathrm{~h}$ darkness cycle with free access to food and water. Heat production and respiratory exchange ratio (RER) were then calculated.

Voluntary activity of each mouse was measured with an optical beam technique (Opto-M3, Columbus Instruments, Columbus, $\mathrm{OH}$, USA) over $24 \mathrm{~h}$ and expressed as 24 -h average activity.

\section{Surgical iBAT removal experiment}

Surgery was performed according to a previously described protocol (Piao et al. 2018) using 8-week-old TG and WT mice. After 10 days of self-recovery, the body weight was tracked for several weeks. Rectal temperature was recorded at the indicated time points (post-exposure 1, 2, 3 and $4 \mathrm{~h}$ ) by using a digital thermometer (Yellow Spring Instruments) during short-term cold exposure $\left(4^{\circ} \mathrm{C}\right)$ with free access to water in order to assess the body temperature maintenance capacity between two groups.

\section{Statistical analysis}

Data are presented as the mean \pm S.E.M. Statistical significance was tested with one-way ANOVA followed by
Tukey's post hoc test or a paired Student's $t$-test. Statistical significance was set at $P<0.05$.

\section{Results}

\section{The miR17-92 cluster is highly enriched in BAT}

Cold exposure is known to enhance BAT thermogenic function and beige cell formation (Barbatelli et al. 2010). To investigate how miRNAs are involved in BAT activation and beige cell formation, we performed RNA sequencing of plasma from mice subjected to cold exposure. As expected, the levels of many circulating miRNAs were significantly altered upon exposure to cold temperatures; all members of the miR17-92 cluster were upregulated in plasma during this process (Fig. 1A). Thus, circulating miR17-92 cluster members may be involved in cold adaptation. We then evaluated this result in adipose tissues after cold exposure; interestingly, in BAT, there were no significant changes in the expression of the miR17-92 cluster (Fig. 1B) despite elevated UCP1 expression after cold exposure (Supplementary Fig. 3A, see section on supplementary materials given at the end of this article). However, this procedure may induce beige cell formation and substantially upregulate the expression of the miR17-92 cluster in subcutaneous (SUB) adipose tissue (Fig. 1C). Furthermore, the expression of the miR17-92 cluster was significantly enriched in BAT compared with SUB and epididymal (EP) adipose tissues (Fig. 1D). These data suggested that the miR17-92 cluster may be involved

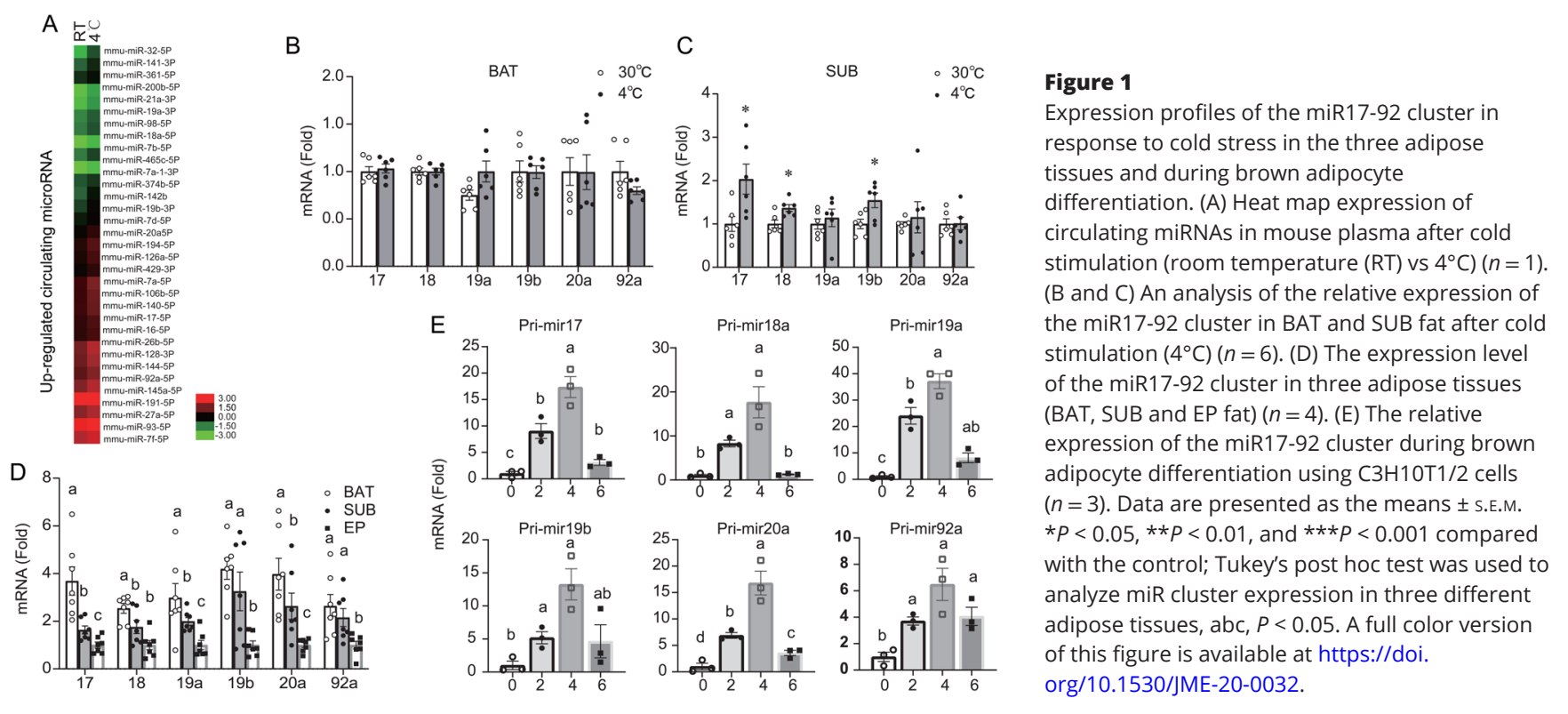


in adaptive thermogenesis during the process of BAT and beige cell formation.

\section{Overexpression of miR17-92 induces the expression of UCP1}

To explore the role of the miR17-92 cluster in brown adipogenesis, we investigated temporal and spatial miR17-92 cluster expression during brown adipogenesis. The expression of the miR17-92 cluster was significantly induced during brown adipogenesis (Fig. 1E). These results led us to hypothesize that the miR17-92 cluster may be involved in brown adipogenesis. To elucidate the role of the miR17-92 cluster during brown adipogenesis, the entire cluster was cloned into a lentiviral expression vector under the control of the cytomegalovirus (CMV) promoter. C3H10T1/2 cells were infected with the lentivirus, and the vehicle was used as a control. After 6 days of brown adipocyte differentiation, we first confirmed that the members of the miR17-92 cluster were all substantially upregulated after overexpression of the miR17-92 cluster (Fig. 2A). Oil Red $\mathrm{O}$ staining indicated that overexpression of the miR17-92 cluster caused higher differentiation efficiency than overexpression of the control (Fig. 2B). In contrast, we found that overexpression of the miR17-92 cluster led to markedly increased expression of UCP1, a BAT-specific marker gene. Moreover, other thermogenic genes, such as CEBP $\beta$, PPAR $\gamma 2$ and PGC $1 \alpha$, were also highly upregulated compared with their levels in the control group (Fig. 2C). In addition, mitochondrial oxidative phosphorylation (OXPHOS) system proteins were also dramatically increased after miR17-92 cluster overexpression (Fig. 2D and E). Consistent with these findings, maximum mitochondrial respiration was significantly increased in the miR17-92 cluster overexpression group, as evaluated using a Seahorse XF24 analyzer (Fig. 2F). To confirm our results, we observed a similar phenotype in another preadipocyte cell line, 3T3-L1, after 10 days of brown adipocyte differentiation (Supplementary Fig. 1A, B, C and D).

These results suggest that the miR17-92 cluster significantly enhances the thermogenic capacity of adipocytes.

\section{Adipose tissue-specific miR17-92 cluster transgenic (TG) mice exhibit reduced adiposity}

The above results demonstrated that the miR17-92 cluster can increase the expression of UCP1 during brown adipogenesis. To determine the physiological role of the miR17-92 cluster, we constructed adipose tissuespecific miR17-92 cluster TG mice using the adiponectin promoter, which specifically expresses the miR17-92 cluster within adipose tissue (Fig. 3A-C) but not muscle (Supplementary Fig. 2A); two members of the circulating miR17-92 cluster were also upregulated in the plasma of the TG mice compared with control mice (Supplementary Fig. 2B). There was reduced adiposity in the TG mice compared with the WT mice (Fig. 3D), while no changes in body weight (Fig. 3E) or blood lipid concentration were found (Supplementary Table 1). Unexpectedly, BAT histology (Fig. 3F), gene expression (Fig. 3G) and protein levels of OXPHOS (Fig. 3H) in the TG mice were nearly identical to those in the WT control mice. In addition, the glucose tolerance test (GTT) revealed no significant differences between the groups (Supplementary Fig. 2C). These results demonstrated that adipose tissue-specific miR17-92 cluster TG mice exhibited reduced adiposity without any changes in BAT function.

\section{The miR17-92 cluster can induce beige cell formation in SUB adipose tissue}

WAT browning could also play an important role in energy expenditure. Therefore, we took a closer look at

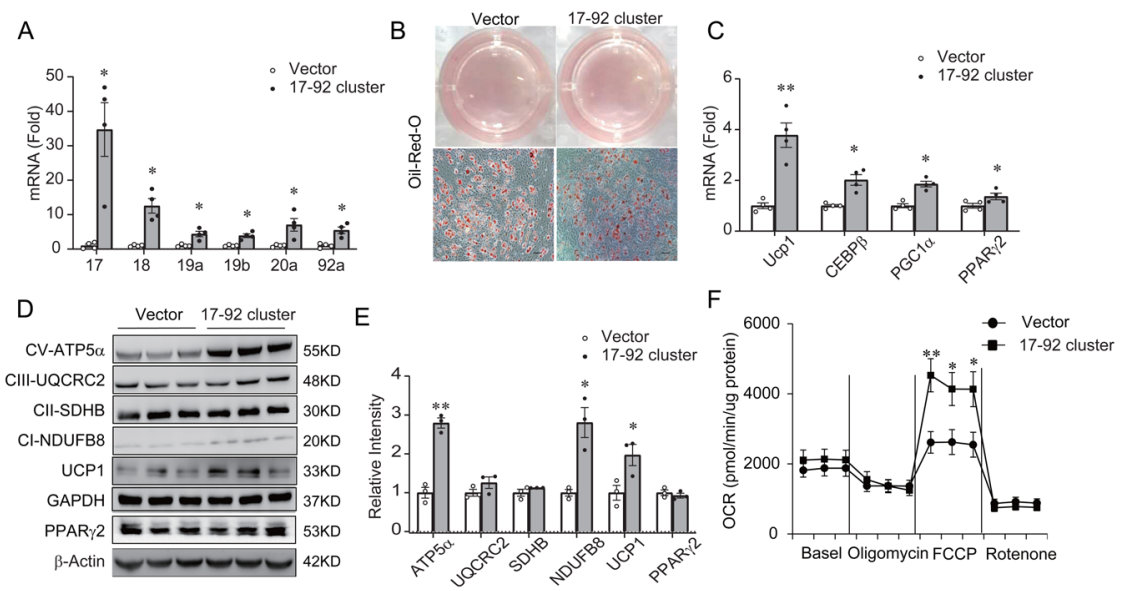

\begin{abstract}
Figure 2
Overexpression of the miR17-92 cluster improved the thermogenic function of $\mathrm{C} 3 \mathrm{H} 10 \mathrm{~T} 1 / 2$ cells after brown differentiation. C3H10T1/2 cells were infected with miR17-92 cluster lentivirus and then harvested on day 6 after brown adipocyte differentiation. (A) The expression level of the miR17-92 cluster $(n=4)$. (B) Oil Red O staining. Scale bars, $100 \mu \mathrm{m}$. (C) The expression of several thermogenic genes $(n=4)$. (D and E) Western blots of UCP1 and OXPHOS ( $n=3)$. (F) Cellular oxygen consumption $(n=6)$. Data are presented as the means \pm S.E.M. $* P<0.05, * * P<0.01$, and $\star \star \star P<0.001$ compared with the control. A full color version of this figure is available at https:// doi.org/10.1530/JME-20-0032.
\end{abstract}

(C) 2020 Society for Endocrinology Published by Bioscientifica Ltd. Printed in Great Britain 

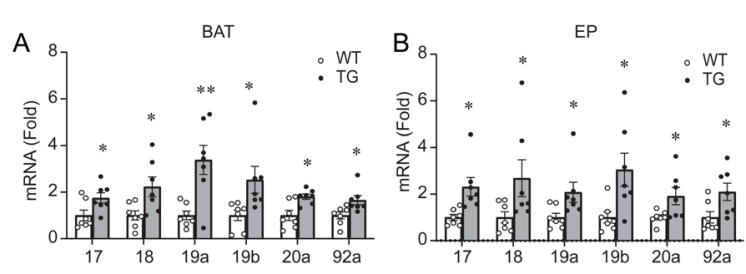

E $0.8 \circ W T$
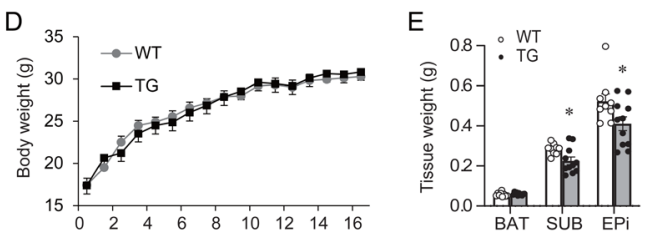

$\mathrm{F}$
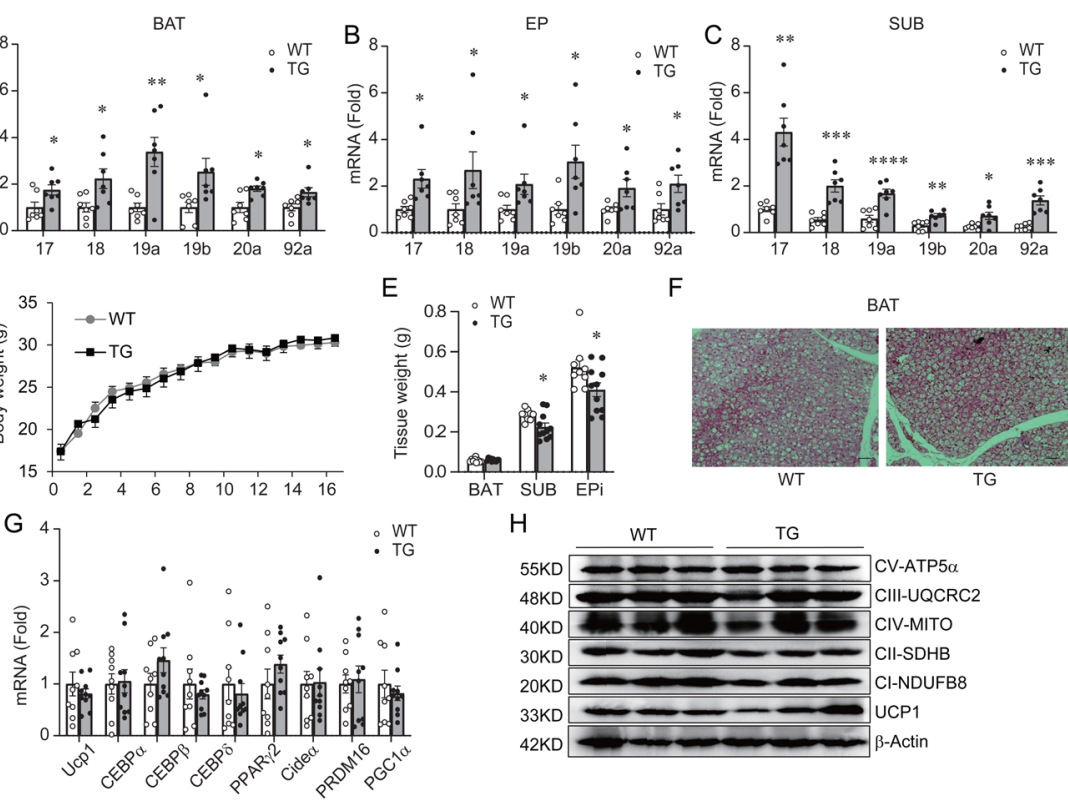

$\mathrm{H}$

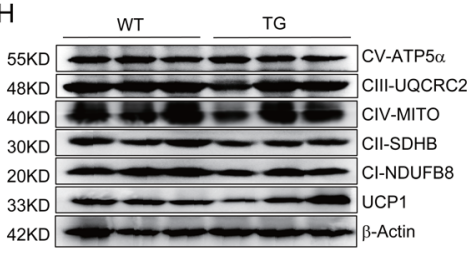

\section{Figure 3}

BAT function was not altered in the miR17-92 cluster transgenic mice. (A-C) The expression level of the miR17-92 cluster in BAT, EP fat and SUB fat $(n=7)$. (D) The body weight of miR17-92 cluster TG mice compared with control mice fed a control diet $(n=9-11)$. (E) The weight of BAT, SUB fat and EP fat $(n=9-11)$. (F) BAT cell morphologies of TG mice. Scale bars, $100 \mu \mathrm{m}$. (G and H) The expression level of oxidative phosphorylationrelated mRNAs $(n=9-10)$ and proteins in BAT $(n=3)$. Data are presented as the means \pm S.E.M. $\star P<0.05, * * P<0.01$, and $* * * P<0.001$ compared with the control. A full color version of this figure is available at https://doi.org/10.1530/ JME-20-0032.
SUB adipose tissue. Interestingly, we revealed that large numbers of multilocular adipocytes accumulated within the SUB adipose tissue of TG mice (Fig. 4A). Furthermore, immunohistochemically staining showed that the SUB adipose tissue from TG mice displayed much higher levels of UCP1 protein expression than the SUB adipose tissue from WT mice (Fig. 4A). Consistent with this finding, we also observed increases in thermogenic (Cidea, PRDM16, $\mathrm{PGC} 1 \alpha, \mathrm{UCP} 1$, and Tfam) and adipogenic (C/EBP $\alpha, \mathrm{C} / \mathrm{EBP} \beta$, $\mathrm{C} / \mathrm{EBP} \delta$, and PPAR $\gamma 2$ ) gene expression in TG SUB adipose tissue compared with the WT adipose tissue (Fig. 4B).
These results were corroborated by the in vitro differentiation of C3H10T1/2 and 3T3-L1 cells. Furthermore, we found that mitochondrial DNA copy number (Fig. 4C) and OXPHOS protein expression (Fig. 4D) were significantly upregulated in TG SUB adipose tissue. These phenomena led to a considerable increase in whole-body energy expenditure (Fig. 4E, F and G) without alterations in physical activity or food intake (Fig. $4 \mathrm{H}$ and I). These results further suggest that increased miR17-92 cluster expression selectively stimulates white fat browning and increases mitochondrial activity in the SUB adipose tissue.

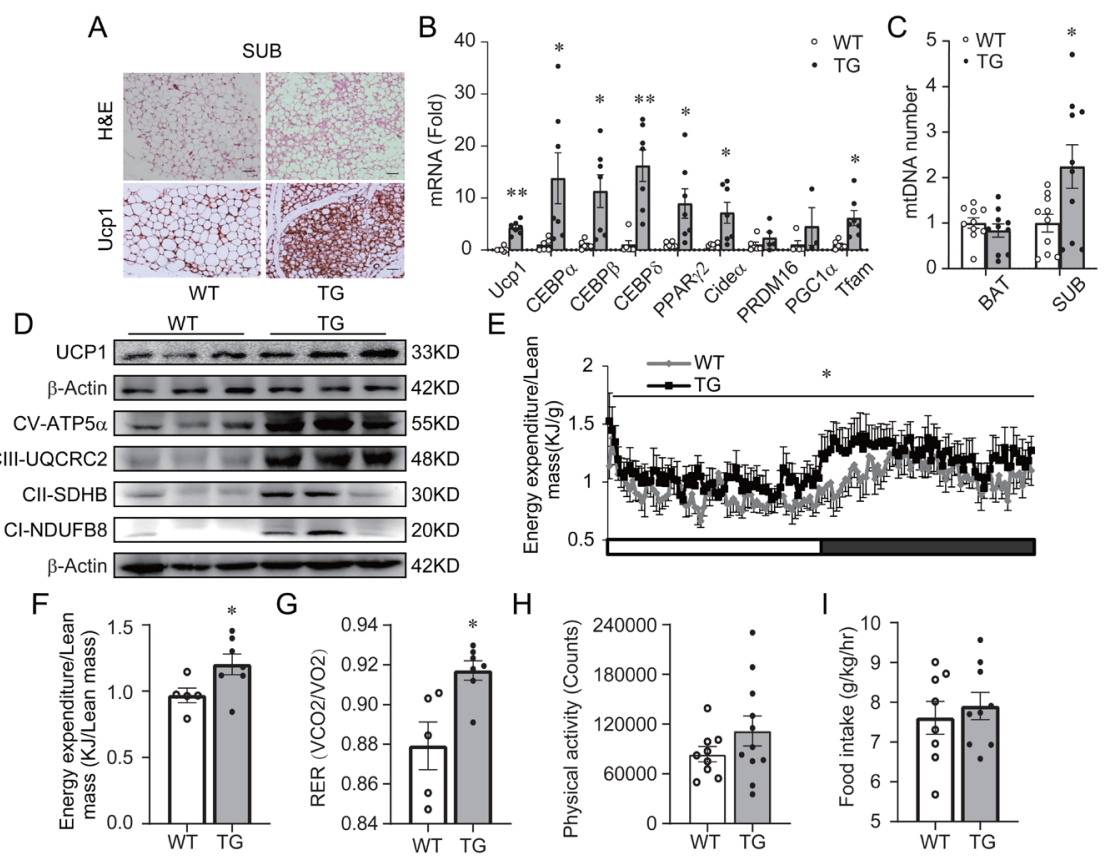

\section{Figure 4}

Overexpression of the miR17-92 cluster activated the browning of SUB fat and increased wholebody energy metabolism. (A) Overexpression of the miR17-92 cluster significantly enhanced the browning of SUB fat, as indicated by H\&E and immunohistochemical (IHC) staining. Scale bars, $100 \mu \mathrm{m}$. (B) The expression of adipogenic and browning-related genes $(n=6-7)$ and (C) mitochondrial DNA copy number were significantly increased in SUB fat after miR17-92 cluster overexpression ( $n=10)$. (D) The overexpression of the miR17-92 cluster increased the levels of the UCP1 and OXPHOS proteins in SUB fat $(n=3)$. (E-G) The miR17-92 cluster TG mice displayed higher whole-body energy expenditure (24 h) than the control group, without changing $(\mathrm{H})$ physical activity and $(\mathrm{I})$ food intake $(n=5-7)$. Data are presented as the means \pm S.E.M. $* P<0.05, * * P<0.01$, and $* * * P<0.001$ compared with the control. A full color version of this figure is available at https://doi.org/10.1530/ JME-20-0032. 
The miR17-92 cluster can promote beige cell formation without iBAT input in TG mice to maintain body temperature under cold exposure

Beige adipocytes are commonly recognized as a positive metabolic organ that could help animals adapt to cold environments. However, in the previous experiment, we did not observe significant differences in body temperature during cold exposure, which is probably due to iBAT interference (Supplementary Fig. 2D). To eliminate the potential role of iBAT in beige fat formation, we removed the iBAT by surgery. After 8 weeks of a normal chow diet under room temperature, the body weights (Fig. 5A), glucose tolerance test (GTT) (Supplementary Fig. 3A), physical activity (Supplementary Fig. 3B), plasma glycerol and TG (Supplementary Fig. 3C and D) concentration of the two groups still did not show a significant difference. When the animals were challenged with cold room $\left(4^{\circ} \mathrm{C}\right)$ exposure for $4 \mathrm{~h}$, the rectal temperatures of TG mice were higher than those of WT mice; however, upon exposure for a longer period $(16 \mathrm{~h})$, the temperature was the same. Furthermore, after 1 week of rest, we repeated the experiment still when the mice were exposure for $4 \mathrm{~h}$, the rectal temperatures of TG mice were higher than those of
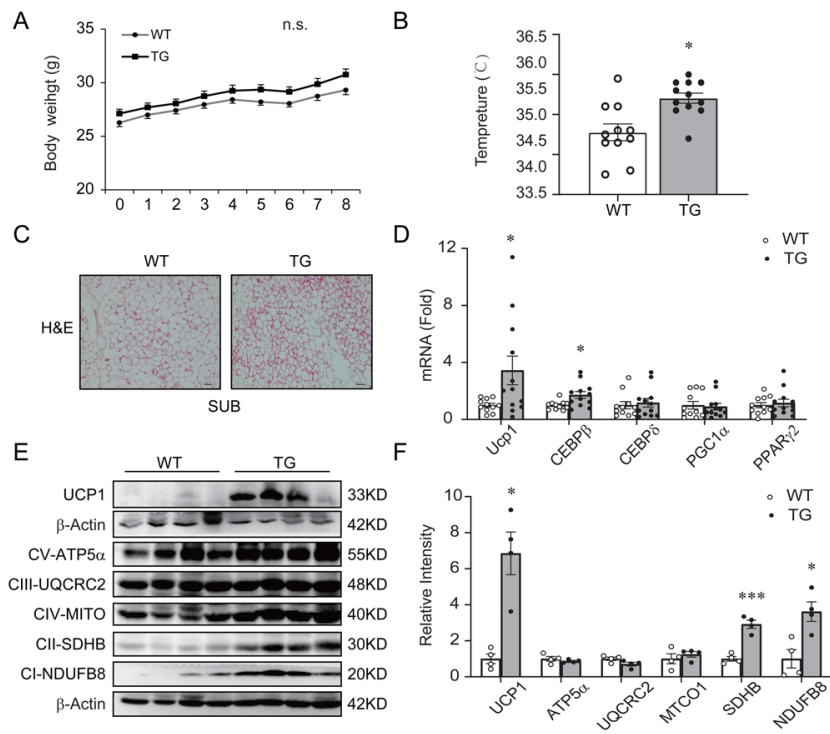

\section{Figure 5}

The miR17-92 cluster promotes thermogenesis upon iBAT removal in TG mice. (A) The body weights of mice who underwent iBAT removal (miR17-92 cluster TG mice and WT mice) fed a chow diet $(n=11-12)$. (B) Rectal temperature after $4 \mathrm{~h}$ of $4^{\circ} \mathrm{C}$ cold exposure $(n=11-12)$. (C) H\&E staining of SUB fat. Scale bars, $100 \mu \mathrm{m}$. (D) The mRNA expression of BAT-related genes $(n=11-12)$. (E and F) Western blot analysis of UCP1 and OXPHOS in SUB fat. All data are normalized to $\beta$-actin. Data are presented as the means \pm S.E.M. $* P<0.05, * \star P<0.01$, and $* \star \star P<0.001$ compared with the control. A full color version of this figure is available at https://doi.org/10.1530/JME-20-0032.
WT mice (Fig. 5B). Then the mice were killed. This finding is in agreement with our hypothesis that without the interference of iBAT, beige fat function can be detected. Accordingly, the H\&E staining showed that more beige fat appeared in the SUB adipose tissue of TG mice than in that of WT mice after cold stimulation (Fig. 5C). Accordingly, the expression levels of thermogenic genes such as UCP1 and adipogenic C/EBP $\beta$ in the SUB adipose tissue of TG mice were significantly elevated compared with those in the SUB adipose tissue of WT mice (Fig. 5D). UCP1 and mitochondrial OXPHOS protein expression were significantly upregulated in the SUB adipose tissue of TG mice compared with that of WT mice (Fig. 5E and F). Overall, the miR17-92 cluster could promote beige cell formation without iBAT input in TG mice to maintain body temperature under cold exposure.

\section{The miR17-92 cluster targets Rb1 in the SUB adipose tissue}

The key question is how the miR17-92 cluster is involved in white fat browning processes in vivo. To identify a potential target of the miR17-92 cluster that is involved in white-to-beige adipocyte conversion, the online programs Target Scan Mouse 6.2 (http://www.targetscan. org/) and miRDB (http://www.mirdb.org/) were used to perform bioinformatic prediction analysis, after which we identified genes related to inhibitors of thermogenesis (Supplementary Fig. 4). Most targets failed to show substantial changes in the SUB fat or BAT of the TG mice (Supplementary Fig. 4B and C) or during brown adipogenesis (Supplementary Fig. 3D). Interestingly, we found that $\mathrm{Rb} 1$ showed a nearly perfect match for recognition sites within the $3^{\prime}$-UTRs of two members of the miR17-92 cluster (miR17 and miR20) (Fig. 6A). In addition, meaningful decreases were observed in both $\mathrm{Rb} 1$ mRNA and protein expression levels in the SUB adipose tissue of TG mice compared with the levels in the SUB adipose tissue of WT control mice (Fig. 6B and C). To assess whether $\mathrm{Rb} 1$ is a direct target of the miR17-92 cluster, we generated a luciferase reporter containing either a WT 3 '-UTR of mouse Rb1 or a variant in which the target sequences were mutated. The mutant reporter exhibited considerably higher activity than the WT reporter upon overexpression of the miR17-92 cluster (Fig. 6D). These results imply that $\mathrm{Rb} 1$ is a target of the miR17-92 cluster. Then, we successfully knocked down Rb1 in C3H10T1/2 cells using shRNA to elucidate the function of $\mathrm{Rb} 1$ in brown adipogenesis (Fig. 6E). Consistent with the results of overexpression of miR17-92, there were no significant 
A

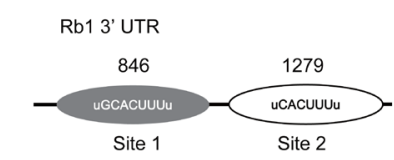

D

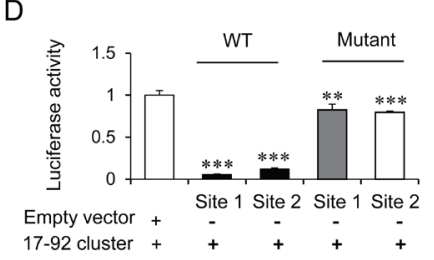

G

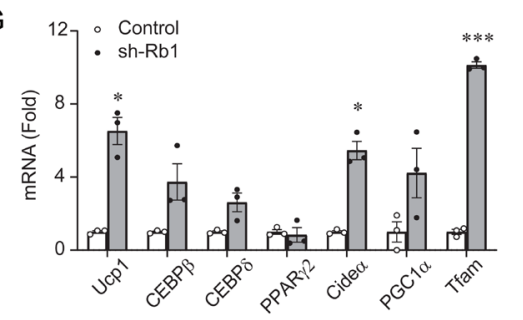

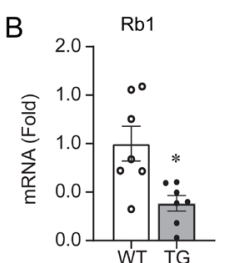

E

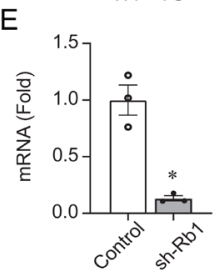

$\mathrm{F}$

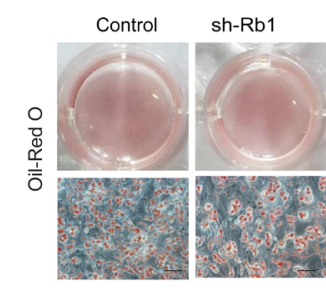

C

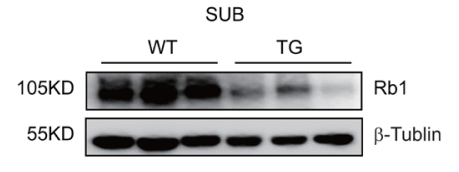

$\mathrm{H}$

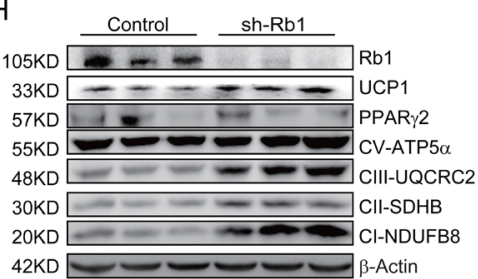

\section{Figure 6}

Overexpression of the miR17-92 cluster enhanced brown adipocyte differentiation by targeting Rb1. (A) Two potential miRNA binding sites were identified in the Rb1 3'-UTR: site 1 and site 2. (B and C) Levels of Rb1 mRNA $(n=7)$ and protein in the SUB fat of TG and WT mice $(n=3)$. (D) Luciferase activity of Rb1 3'-UTR. HEK293T cells were transfected with or without lentivirus, including the miR17-92 cluster, together with a wild-type (WT) or mutant (Mut) Rb1 3'-UTR inserted downstream of firefly luciferase $(n=4)$. (E) The expression level of Rb1 after shRNA knockdown ( $n=3$ ). (F) Oil Red O staining of Rb1 knockdown after brown adipocyte differentiation $(n=3-4)$. Scale bars, $100 \mu \mathrm{m}$. (G and H) The expression levels of thermogenic and oxidative phosphorylation-related mRNAs and proteins $(n=3)$. Data are presented as the means \pm S.E.M. $\star P<0.05, * * P<0.01$, and $* * * P<0.001$ compared with the control. A full color version of this figure is available at https://doi.org/10.1530/ JME-20-0032. differences in differentiation efficiency between the two groups as assessed by Oil Red O staining (Fig. 6F). Similarly, we observed significant increases in the expression of thermogenic genes (UCP1, Cidea, and TFAM) and levels of OXPHOS proteins after Rb1 knockdown (Fig. 6G and $\mathrm{H})$. Based on these results, the miR17-92 cluster targets Rb1 to enhance white fat browning.

\section{Discussion}

Recently beige cells have attracted increasing attention due to their important functions in metabolism. A notable feature of beige cells is that they can be induced by various environmental cues, such as chronic cold exposure (Barbatelli et al. 2010), exercise (Bostrom et al. 2012) and many transcription factors, including PPAR, PRDM16, CEBP $\beta$, HOXC8, RIP140, and Zic1 (Zhang et al. 2015, Inagaki et al. 2016). Circulating miRNAs, also known as extracellular miRNAs (ECmiRNAs), have been detected in various biological fluids and cell culture media (Valadi et al. 2007, Chen et al. 2008, Chim et al. 2008, Hunter et al. 2008, Mitchell et al. 2008, Arroyo et al. 2011, da Silveira et al. 2012, Sang et al. 2013, Sohel et al. 2013, Noferesti et al. 2015). miRNAs are regulated by various cytokines (Zhu et al. 2013, Hale et al. 2014, Krysan et al. 2014, Hamrick 2017) and have been linked to organ-organ interactions for metabolic control. miR378 promoted adipogenesis and was downregulated in cardiac myocytes during heart failure (Rottiers \& Naar 2012, Ganesan et al. 2013). Furthermore, miR144 can regulate cholesterol export in macrophages and HDL biogenesis by reducing ABCA1 expression (Ramirez et al. 2013). These miRNAs may be regarded as metabolic mediators of multiorgan crosstalk and may be involved in the pathogenesis of endocrine-associated metabolic diseases. To the best of our knowledge, we are the first to verify that the miR17-92 cluster stimulated WAT browning without altering BAT activity in mice. The miR17-92 cluster showed different functions in BAT and WAT depending on various target genes. The miR17-92 cluster was highly enriched in BAT compared with other WAT, and cold exposure induced the upregulation of the miR17-92 cluster. Thus, the miR17-92 cluster may play an important role in regulating thermogenesis.

According to previous reports, the miRNAs enriched in brown fat may play a critical role in thermogenesis and energy expenditure. For example, miR378 controls classic brown fat expansion to counteract obesity, and miR193b-365 is essential for brown fat differentiation. In accord with these observations, we identified the miR17-92 cluster as highly expressed in brown fat compared with other WATs. Surprisingly, in our study, no differences in body weight or BAT function in miR17-92 TG mice were observed. Interestingly, the miR17-92 cluster upregulates the UCP1 gene, a key factor in thermogenic function, and increases mitochondrial number, fatty acid oxidation level and $\mathrm{O}_{2}$ consumption in subcutaneous fat without changing food intake or physical activity. These results indicate that the miR17-92 cluster may prompt beige fat formation in WAT. Accordingly, miR17-92 cluster 
overexpression could stimulate the differentiation of white adipocytes into beige cells and UCP1 expression in the 3T3-L1 preadipocyte cell line.

Unlike rodents with classic BAT, only beige fat but no BAT has been identified in adult humans (Wu et al. 2012). As shown in our recent study, interscapular BAT (iBAT) removal significantly enhances WAT browning due to compensatory sympathetic WAT input (Piao et al. 2018). Furthermore, WAT browning plays an important role in whole-body energy metabolism during cold acclimation, even without iBAT (Piao et al. 2018). In parallel, our results also show that the miR17-92 cluster promotes beige fat formation in WAT, which enhances whole-body oxygen consumption. In agreement with this notion, beige fat formation is further enhanced in TG mice after removal of iBAT. Therefore, the induction of beige cells within WAT is a more feasible strategy to maintain better body fat content and a higher metabolic rate.

In a previous study, the miR17-92 cluster induced differentiation and increased triglyceride accumulation in 3T3-L1 cells by targeting Rb2/p130 (Wang et al. 2008). It has been reported that $\mathrm{P} 107$ and $\mathrm{P} 130$ regulate adipogenesis in 3T3-L1 preadipocytes (Richon et al. 1997). In addition, it has also been shown that P107 and $\mathrm{Rb} 1$ exert synergistic effects during browning (Scime et al. 2005). Furthermore, Rb KO mice exhibited decreased body weight because of increased energy expenditure (Dali-Youcef et al. 2007). In our study, increasing energy expenditure in miR17-92 TG mice did not cause body weight changes. This difference is likely because $\mathrm{Rb} \mathrm{KO}$ mice exhibited activated BAT in addition to the browning of WAT.

In conclusion, to the best of our knowledge, our study is the first to show that the miR17-92 cluster stimulates WAT browning without altering BAT activity and function in mice. Overexpression of the miR17-92 cluster in C3H10T1/2 cells induced the expression of BAT thermogenic genes, especially UCP1, compared with the control cells. Accordingly, adipose tissue-specific miR17-92 cluster TG mice exhibited WAT browning, increased energy expenditure and decreased adiposity by targeting $\mathrm{Rb} 1$, a beige cell repressor in WAT. Thus, the miR17-92 cluster may play an important role in thermogenic regulation.

\section{Supplementary materials}

This is linked to the online version of the paper at https://doi.org/10.1530/ JME-20-0032.

\section{Declaration of interest}

The authors declare that there is no conflict of interest that could be perceived as prejudicing the impartiality of the research reported.

\section{Funding}

This work was supported by the National Key Research and Development Program of China (grant number 2017YFC1001001, 2017), grants from the National Natural Science Foundation of China (grant numbers 81770834 , 2018 and 81370951, 2014) and the Strategic Priority Research Program (grant number XDB13030000, 2014) from the Chinese Academy of Sciences.

\section{Data availability}

The data used to support the findings of this study are available from the corresponding author upon request (contact details: Wanzhu Jin, 1 Beichen West Road, Chaoyang District, Beijing 100101, P. R. China, email: jinw@ioz. ac.cn).

\section{Author contribution statement}

Dr Wanzhu Jin and Xiaomeng Liu are the guarantors of this work and thus have full access to all the data in the study and take responsibility for the integrity of the data and the accuracy of the data analysis. H Y Y, Z H L and J W Z wrote the manuscript. HYY performed the histological analysis, Western blot experiments, gene expression analyses and cell-based experiments. $\mathrm{Z} \mathrm{H} \mathrm{L} \mathrm{performed} \mathrm{the} \mathrm{energy} \mathrm{metabolism} \mathrm{assays.} \mathrm{Z} \mathrm{H} \mathrm{L,} \mathrm{D} \mathrm{M,}$ Z L, L J, Y R C and Z H Q performed the animal experiments.

\section{References}

Arroyo JD, Chevillet JR, Kroh EM, Ruf IK, Pritchard CC, Gibson DF, Mitchell PS, Bennett CF, Pogosova-Agadjanyan EL, Stirewalt DL, et al. 2011 Argonaute 2 complexes carry a population of circulating microRNAs independent of vesicles in human plasma. PNAS $\mathbf{1 0 8}$ 5003-5008. (https://doi.org/10.1073/pnas.1019055108)

Barbatelli G, Murano I, Madsen L, Hao Q, Jimenez M, Kristiansen K, Giacobino JP, De Matteis R \& Cinti S 2010 The emergence of coldinduced brown adipocytes in mouse white fat depots is determined predominantly by white to brown adipocyte transdifferentiation. American Journal of Physiology: Endocrinology and Metabolism 298 E1244-E1253. (https://doi.org/10.1152/ajpendo.00600.2009)

Bostrom P, Wu J, Jedrychowski MP, Korde A, Ye L, Lo JC, Rasbach KA, Bostrom EA, Choi JH, Long JZ, et al. 2012 A PGC1-alpha-dependent myokine that drives brown-fat-like development of white fat and thermogenesis. Nature 481 463-468. (https://doi.org/10.1038/ nature10777)

Chen X, Ba Y, Ma L, Cai X, Yin Y, Wang K, Guo J, Zhang Y, Chen J, Guo X, et al. 2008 Characterization of microRNAs in serum: a novel class of biomarkers for diagnosis of cancer and other diseases. Cell Research 18 997-1006. (https://doi.org/10.1038/cr.2008.282)

Chen Y, Siegel F, Kipschull S, Haas B, Frohlich H, Meister G \& Pfeifer A 2013 miR-155 regulates differentiation of brown and beige adipocytes via a bistable circuit. Nature Communications 41769. (https://doi.org/10.1038/ncomms2742)

Chim SS, Shing TK, Hung EC, Leung TY, Lau TK, Chiu RW \& Lo YM 2008 Detection and characterization of placental microRNAs in maternal plasma. Clinical Chemistry 54 482-490. (https://doi. org/10.1373/clinchem.2007.097972)

Classon M \& Dyson N 2001 p107 and p130: versatile proteins with interesting pockets. Experimental Cell Research 264 135-147. (https:// doi.org/10.1006/excr.2000.5135) 
Cypess AM, Lehman S, Williams G, Tal I, Rodman D, Goldfine AB, Kuo FC, Palmer EL, Tseng YH, Doria A, et al. 2009 Identification and importance of brown adipose tissue in adult humans. New England Journal of Medicine 360 1509-1517. (https://doi.org/10.1056/ NEJMoa0810780)

Da Silveira JC, Veeramachaneni DN, Winger QA, Carnevale EM \& Bouma GJ 2012 Cell-secreted vesicles in equine ovarian follicular fluid contain miRNAs and proteins: a possible new form of cell communication within the ovarian follicle. Biology of Reproduction $\mathbf{8 6}$ 71. (https://doi.org/10.1095/biolreprod.111.093252)

Dali-Youcef N, Mataki C, Coste A, Messaddeq N, Giroud S, Blanc S, Koehl C, Champy MF, Chambon P, Fajas L, et al. 2007 Adipose tissue-specific inactivation of the retinoblastoma protein protects against diabesity because of increased energy expenditure. PNAS 104 10703-10708. (https://doi.org/10.1073/pnas.0611568104)

Ganesan J, Ramanujam D, Sassi Y, Ahles A, Jentzsch C, Werfel S, Leierseder S, Loyer X, Giacca M, Zentilin L, et al. 2013 miR-378 controls cardiac hypertrophy by combined repression of mitogenactivated protein kinase pathway factors. Circulation 127 2097-2106. (https://doi.org/10.1161/CIRCULATIONAHA.112.000882)

Hale A, Lee C, Annis S, Min PK, Pande R, Creager MA, Julian CG, Moore LG, Mitsialis SA, Hwang SJ, et al. 2014 An Argonaute 2 switch regulates circulating miR-210 to coordinate hypoxic adaptation across cells. Biochimica et Biophysica Acta 1843 2528-2542. (https:// doi.org/10.1016/j.bbamcr.2014.06.012)

Hamrick MW 2017 Role of the cytokine-like hormone leptin in musclebone crosstalk with aging. Journal of Bone Metabolism 24 1-8. (https://doi.org/10.11005/jbm.2017.24.1.1)

Hunter MP, Ismail N, Zhang X, Aguda BD, Lee EJ, Yu L, Xiao T, Schafer J, Lee ML, Schmittgen TD, et al. 2008 Detection of microRNA expression in human peripheral blood microvesicles. PLOS ONE 3 e3694. (https://doi.org/10.1371/journal.pone.0003694)

Inagaki T, Sakai J \& Kajimura S 2016 Transcriptional and epigenetic control of brown and beige adipose cell fate and function. Nature Reviews: Molecular Cell Biology 17 480-495. (https://doi.org/10.1038/ nrm.2016.62)

Jordan SD, Kruger M, Willmes DM, Redemann N, Wunderlich FT, Bronneke HS, Merkwirth C, Kashkar H, Olkkonen VM, Bottger T, et al. 2011 Obesity-induced overexpression of miRNA-143 inhibits insulin-stimulated AKT activation and impairs glucose metabolism. Nature Cell Biology 13 434-446. (https://doi.org/10.1038/ncb2211)

Kalinovich AV, De Jong JM, Cannon B \& Nedergaard J 2017 UCP1 in adipose tissues: two steps to full browning. Biochimie 134 127-137. (https://doi.org/10.1016/j.biochi.2017.01.007)

Krysan K, Kusko R, Grogan T, O’Hearn J, Reckamp KL, Walser TC, Garon EB, Lenburg ME, Sharma S, Spira AE, et al. 2014 PGE2-driven expression of c-Myc and oncomiR-17-92 contributes to apoptosis resistance in NSCLC. Molecular Cancer Research 12 765-774. (https:// doi.org/10.1158/1541-7786.MCR-13-0377)

Lowell BB \& Spiegelman BM 2000 Towards a molecular understanding of adaptive thermogenesis. Nature $\mathbf{4 0 4} 652-660$. (https://doi. org/10.1038/35007527)

Mendell JT 2008 miRiad roles for the miR-17-92 cluster in development and disease. Cell 133 217-222. (https://doi.org/10.1016/j. cell.2008.04.001)

Mercader J, Ribot J, Murano I, Feddersen S, Cinti S, Madsen L, Kristiansen K, Bonet ML \& Palou A 2009 Haploinsufficiency of the retinoblastoma protein gene reduces diet-induced obesity, insulin resistance, and hepatosteatosis in mice. American Journal of Physiology: Endocrinology and Metabolism 297 E184-E193. (https://doi. org/10.1152/ajpendo.00163.2009)

Mitchell PS, Parkin RK, Kroh EM, Fritz BR, Wyman SK, PogosovaAgadjanyan EL, Peterson A, Noteboom J, O’Briant KC, Allen A, et al. 2008 Circulating microRNAs as stable blood-based markers for cancer detection. PNAS 105 10513-10518. (https://doi.org/10.1073/ pnas.0804549105)
Mori MA, Thomou T, Boucher J, Lee KY, Lallukka S, Kim JK, Torriani M, Yki-Jarvinen H, Grinspoon SK, Cypess AM, et al. 2014 Altered miRNA processing disrupts brown/white adipocyte determination and associates with lipodystrophy. Journal of Clinical Investigation 124 3339-3351. (https://doi.org/10.1172/JCI73468)

Noferesti SS, Sohel MM, Hoelker M, Salilew-Wondim D, Tholen E, Looft C, Rings F, Neuhoff C, Schellander K \& Tesfaye D 2015 Controlled ovarian hyperstimulation induced changes in the expression of circulatory miRNA in bovine follicular fluid and blood plasma. Journal of Ovarian Research 8 81. (https://doi.org/10.1186/ s13048-015-0208-5)

Petrovic N, Walden TB, Shabalina IG, Timmons JA, Cannon B \& Nedergaard J 2010 Chronic peroxisome proliferator-activated receptor gamma (PPARgamma) activation of epididymally derived white adipocyte cultures reveals a population of thermogenically competent, UCP1-containing adipocytes molecularly distinct from classic brown adipocytes. Journal of Biological Chemistry 285 7153-7164. (https://doi.org/10.1074/jbc.M109.053942)

Piao ZY, Zhai BQ, Jiang XX, Dong M, Yan CG, Lin J \& Jin WZ 2018 Reduced adiposity by compensatory WAT browning upon iBAT removal in mice. Biochemical and Biophysical Research Communications 501 807-813. (https://doi.org/10.1016/j.bbrc.2018.05.089)

Ramirez CM, Rotllan N, Vlassov AV, Davalos A, Li M, Goedeke L, Aranda JF, Cirera-Salinas D, Araldi E, Salerno A, et al. 2013 Control of cholesterol metabolism and plasma high-density lipoprotein levels by microRNA-144. Circulation Research 112 1592-1601. (https://doi. org/10.1161/CIRCRESAHA.112.300626)

Richon VM, Lyle RE \& Mcgehee Jr RE 1997 Regulation and expression of retinoblastoma proteins p107 and p130 during 3T3-L1 adipocyte differentiation. Journal of Biological Chemistry 272 10117-10124. (https://doi.org/10.1074/jbc.272.15.10117)

Rosen ED \& Spiegelman BM 2014 What we talk about when we talk about fat. Cell 156 20-44. (https://doi.org/10.1016/j.cell.2013.12.012)

Rottiers V \& Naar AM 2012 MicroRNAs in metabolism and metabolic disorders. Nature Reviews: Molecular Cell Biology 13 239-250. (https:// doi.org/10.1038/nrm3313)

Saito M, Okamatsu-Ogura Y, Matsushita M, Watanabe K, Yoneshiro T, Nio-Kobayashi J, Iwanaga T, Miyagawa M, Kameya T, Nakada K, et al. 2009 High incidence of metabolically active brown adipose tissue in healthy adult humans: effects of cold exposure and adiposity. Diabetes 58 1526-1531. (https://doi.org/10.2337/db09-0530)

Sang Q, Yao Z, Wang H, Feng R, Wang H, Zhao X, Xing Q, Jin L, He L, Wu L, et al. 2013 Identification of microRNAs in human follicular fluid: characterization of microRNAs that govern steroidogenesis in vitro and are associated with polycystic ovary syndrome in vivo. Journal of Clinical Endocrinology and Metabolism 98 3068-3079. (https://doi.org/10.1210/jc.2013-1715)

Scime A, Grenier G, Huh MS, Gillespie MA, Bevilacqua L, Harper ME \& Rudnicki MA $2005 \mathrm{Rb}$ and p107 regulate preadipocyte differentiation into white versus brown fat through repression of PGC-1alpha. Cell Metabolism 2 283-295. (https://doi.org/10.1016/j.cmet.2005.10.002)

Sohel MM, Hoelker M, Noferesti SS, Salilew-Wondim D, Tholen E, Looft C, Rings F, Uddin MJ, Spencer TE, Schellander K, et al. 2013 Exosomal and non-exosomal transport of extra-cellular microRNAs in follicular fluid: implications for bovine oocyte developmental competence. PLoS ONE 8 e78505. (https://doi.org/10.1371/journal.pone.0078505)

Sun L \& Trajkovski M 2014 miR-27 orchestrates the transcriptional regulation of brown adipogenesis. Metabolism: Clinical and Experimental 63 272-282. (https://doi.org/10.1016/j. metabol.2013.10.004)

Tanzer A \& Stadler PF 2004 Molecular evolution of a microRNA cluster. Journal of Molecular Biology 339 327-335. (https://doi.org/10.1016/j. jmb.2004.03.065)

Trajkovski M, Ahmed K, Esau CC \& Stoffel M 2012 MyomiR-133 regulates brown fat differentiation through Prdm16. Nature Cell Biology 14 1330-1335. (https://doi.org/10.1038/ncb2612) 
Valadi H, Ekstrom K, Bossios A, Sjostrand M, Lee JJ \& Lotvall JO 2007 Exosome-mediated transfer of mRNAs and microRNAs is a novel mechanism of genetic exchange between cells. Nature Cell Biology 9 654-659. (https://doi.org/10.1038/ncb1596)

Wang Q, Li YC, Wang J, Kong J, Qi Y, Quigg RJ \& Li X 2008 miR-17-92 cluster accelerates adipocyte differentiation by negatively regulating tumor-suppressor Rb2/p130. PNAS 105 2889-2894. (https://doi. org/10.1073/pnas.0800178105)

Wang ZV, Deng Y, Wang QA, Sun K \& Scherer PE 2010 Identification and characterization of a promoter cassette conferring adipocytespecific gene expression. Endocrinology 151 2933-2939. (https://doi. org/10.1210/en.2010-0136)

Wang J, Liu R, Wang F, Hong J, Li X, Chen M, Ke Y, Zhang X, Ma Q, Wang R, et al. 2013 Ablation of LGR4 promotes energy expenditure by driving white-to-brown fat switch. Nature Cell Biology $\mathbf{1 5}$ 1455-1463. (https://doi.org/10.1038/ncb2867)

Wu J, Bostrom P, Sparks LM, Ye L, Choi JH, Giang AH, Khandekar M, Virtanen KA, Nuutila P, Schaart G, et al. 2012 Beige adipocytes are a distinct type of thermogenic fat cell in mouse and human. Cell $\mathbf{1 5 0}$ 366-376. (https://doi.org/10.1016/j.cell.2012.05.016)
Yin H, Pasut A, Soleimani VD, Bentzinger CF, Antoun G, Thorn S, Seale P, Fernando P, Van Ijcken W, Grosveld F, et al. 2013 MicroRNA-133 controls brown adipose determination in skeletal muscle satellite cells by targeting Prdm16. Cell Metabolism 17 210-224.

Yuan X, Wei G, You Y, Huang Y, Lee HJ, Dong M, Lin J, Hu T, Zhang H, Zhang C, et al. 2017 Rutin ameliorates obesity through brown fat activation. FASEB Journal 31 333-345. (https://doi.org/10.1096/ fj.201600459RR)

Zhang HL, Huang YY, Lee HJ \& Jin WZ 2015 Zic1 negatively regulates brown adipogenesis in C3H10T1/2 cells. Science Bulletin 60 1033-1035. (https://doi.org/10.1007/s11434-015-0797-9)

Zhao S, Jang C, Liu J, Uehara K, Gilbert M, Izzo L, Zeng X, Trefely S, Fernandez S, Carrer A, et al. 2020 Dietary fructose feeds hepatic lipogenesis via microbiota-derived acetate. Nature 579 586-591. (https://doi.org/10.1038/s41586-020-2101-7)

Zhu L, Shi C, Ji C, Xu G, Chen L, Yang L, Fu Z, Cui X, Lu Y \& Guo X 2013 FFAs and adipokine-mediated regulation of hsa-miR-143 expression in human adipocytes. Molecular Biology Reports 40 5669-5675. (https://doi.org/10.1007/s11033-013-2668-2)

Received in final form 22 July 2020

Accepted 4 August 2020

Accepted Manuscript published online 4 August 2020
(C) 2020 Society for Endocrinology Published by Bioscientifica Ltd. Printed in Great Britain 